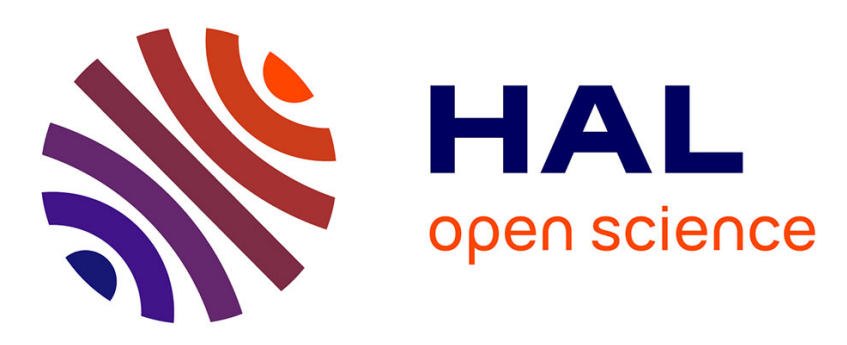

\title{
Inverse Algorithms for the GPR Assessment of Concrete Structures
}

Xisto Lucas Travassos, Douglas A. G. Vieira, Nathan Ida, Christian Vollaire, Alain Nicolas

\section{- To cite this version:}

Xisto Lucas Travassos, Douglas A. G. Vieira, Nathan Ida, Christian Vollaire, Alain Nicolas. Inverse Algorithms for the GPR Assessment of Concrete Structures. IEEE Transactions on Magnetics, 2008, 44 (6), pp.994-997. 10.1109/TMAG.2007.916661 . hal-00358856

\section{HAL Id: hal-00358856 https://hal.science/hal-00358856}

Submitted on 7 Apr 2009

HAL is a multi-disciplinary open access archive for the deposit and dissemination of scientific research documents, whether they are published or not. The documents may come from teaching and research institutions in France or abroad, or from public or private research centers.
L'archive ouverte pluridisciplinaire HAL, est destinée au dépôt et à la diffusion de documents scientifiques de niveau recherche, publiés ou non, émanant des établissements d'enseignement et de recherche français ou étrangers, des laboratoires publics ou privés. 


\title{
Inverse Algorithms for the GPR Assessment of Concrete Structures
}

\author{
X. Lucas Travassos ${ }^{1}$, Jr., D. A. G. Vieira ${ }^{2}$, Nathan Ida $^{3}$, Fellow, IEEE, Christian Vollaire ${ }^{4}$, and Alain Nicolas ${ }^{4}$ \\ ${ }^{1}$ SENAI-CIMATEC, Salvador, Brazil \\ ${ }^{2}$ Federal University of Minas Gerais, Belo Horizonte CEP 31270-901, Brazil \\ ${ }^{3}$ Department of Electrical and Computer Engineering, The University of Akron, Akron, OH 44325 USA \\ ${ }^{4}$ Ecole Centrale de Lyon (ECL), Lyon 69006, France
}

\begin{abstract}
This paper investigates the characterization of inclusions in concrete structures, including the number of inclusions, their geometries, and electromagnetic properties. To solve this problem, a two phase algorithm that combines matched-filter-based reverse-time (MFBRT) migration algorithm with the particle swarm optimization (PSO) is employed. The first phase runs the MFBRT that can, robustly, define the number of inclusions and their centers; however, it cannot define the inclusion geometry and electromagnetic properties. Given the results obtained in the first phase, the PSO is launched in the second phase, in a parametric approach, to define the radii of the inclusions and their properties. Three types of inclusions were considered, water, air, and conductor. Results considering a nonhomogenous host medium having from one to three inclusions are presented showing the effectiveness of the proposed approach.
\end{abstract}

Index Terms-Finite-difference time domain (FDTD), inverse scattering, migration algorithms, particle swarm optimization.

\section{INTRODUCTION}

$\mathbf{I}$ MAGE classification is a commonly pursued areas in diverse fields such as military, security systems, health monitoring, and biomedical engineering. This is due to the fact that it is necessary to eliminate the risk of human misinterpretation by using a machine. The main idea is to obtain information by processing data obtained from sensors. Such machines can substantially reduce the time employed for interpretation and improve accuracy of making decisions by humans operators. The major technical problem when working with imaging algorithms is the large variation in the inspected target signature due to environmental conditions, geometric variations, noise, and sensors' characteristics.

In the past, various imaging or inversion techniques have been developed to refocus the scattered signals back to their true spatial location. Most of them were based on the numerical inversion of the integral equations. All these techniques are characterized by a high level of complexity and, even if the accuracy of the solution is good they require a significant computational burden.

This paper combines two algorithms, in a two-phase approach, for processing ground penetrating radar (GPR) data by means of linear inverse scattering. First, the definition of the number of inclusions and their centers, is done using a matched-filter-based reverse-time (MFBRT) migration algorithm.

This is a well developed technique that can deliver robust results. Nevertheless, it cannot be used to define the inclusion radius and electromagnetic properties. After that, the second phase is launched by using the particle swarm optimization (PSO), in a parametric approach, to the define the unknown variables. The combination of both algorithms in the proposed framework takes advantage of their main strengths minimizing their shortcomings. The MFBRT alone would not be capable to deliver a complete description of the inclusions but it helps

Digital Object Identifier 10.1109/TMAG.2007.916661 in decreasing the PSO computational burden as the number of optimization variables is halved. This paper investigates this combination considering a nonhomogenous host medium with many inclusions of different radii and properties. The inclusions considered are the following: water, air, or conductor.

The paper is structured as follows. First, the inverse scattering problem to characterization of inclusion in a nonhomogenous concrete medium is described. After the nonparametric and parametric approach to solve the problem are presented. It is shown that the parametric approach can decrease its search space by using the information of the nonparametric one and it is the main contribution of the paper. Finally, some results are presented show the effectiveness of the proposed ideas.

\section{INVERSE SCATTERING PROBLEM}

To predict the result of a measurement requires a model of the system under investigation and a physical theory linking the parameters of the model to the parameters being measured.

This prediction of observations, given the values of the parameters defining the model, constitutes the forward problem. The inverse problem consists in using the results of actual observations to infer the values of the parameters characterizing the system under investigation.

Estimating inclusions characteristics using measured signals obtained by scanning antennas is known as an ill-posed inverse problem. Electromagnetic wave propagation inverse problems are typically ill-posed, as opposed to the well-posed problems more typical when modeling physical situations where the model parameters or material properties are known.

For the radar assessment of concrete, the objective is to determine a finite number of parameters. The parameters needed to characterize inclusions in a dielectric slab are found by identifying electrical (permittivity and conductivity) and geometrical (depth and radii) properties. Such a problem is called discrete inverse problem or parameter estimation problem. In general, this is a difficult problem because the obtained information is not sufficient for estimation, which requires some a priori information about the inclusions. 


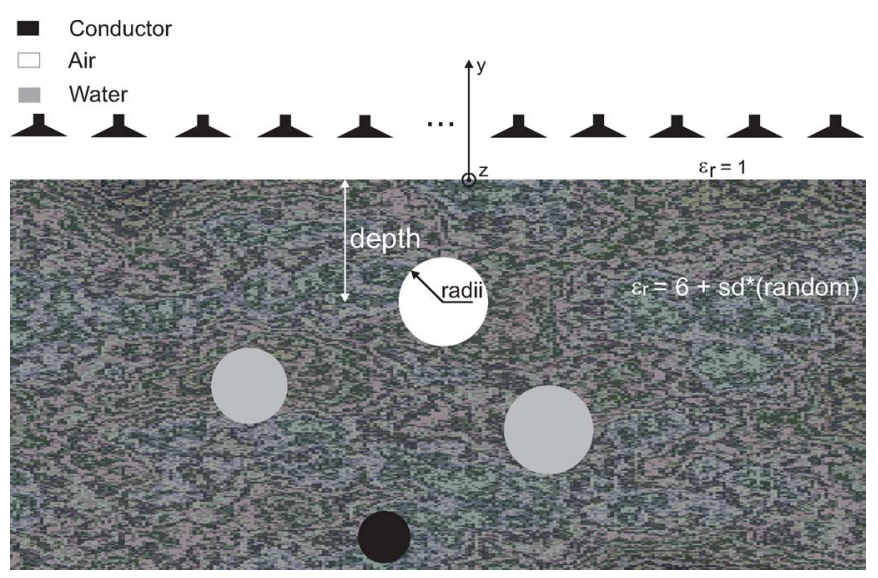

Fig. 1. Configuration consisting of circular cylinders located in a nonhomogeneous dielectric. The problem is composed of a set of inclusions with different properties. The aim is, having the scattered wave, to define the inclusions geometry and properties.

On the other hand, a large number of data can bring the problem to instability. The problem to be solved in this work is to find and characterize inclusions in a nonhomogeneous medium (see Fig. 1). The final result must find the number of inclusions their radius and physical characteristics.

The dielectric medium in this problem is simulated with electrical characteristics of concrete [4] with a mean relative electrical permittivity value of 6 and standard deviation 0.25 , i.e, a nonhomogeneous medium. The finite-difference time domain (FDTD) forward solver is used with a source defined by a differentiated Gaussian pulse with a center frequency of $f=900 \mathrm{MHz}$ and bandwidth between 0.3 and $2 \mathrm{GHz}$ with antennas being simulated as dipoles of infinite extension in the $z$ direction. A sampling interval of 20 ps was chosen to meet the stability criterion of the FDTD, while 1500 samples were collected for each trace corresponding to a time interval of $30 \mathrm{~ns}$. In order to control the numerical dispersion and provide a good discretization for the inclusions, the spatial steps were chosen as $\Delta x=\Delta y=12 \mathrm{~mm}$. The aim of this problem is, given an incident wave, and a scattered wave, to determine the physical and spatial characteristics of the inclusion.

Nonparametric algorithms can also be used to solve this problem. Usually they are complex to implement, but they can solve inverse problems faster than parametric algorithms because it is not necessary to iteratively evaluate the function. Migration algorithms belong to nonparametric algorithms. Migration is an imaging technique commonly used for seismic prospecting. However, it can be applied to general media and targets. In this paper, a migration algorithm for vector waves was implemented based on the idea of matched-filter [1]. Even though this is a robust method for solving the aforementioned problem, it can only defines the number of inclusions and their center. This method will be described in the next section.

Another possible technique to solve this problem is the use of parametric algorithms which are based on optimization algorithms. This kind of algorithms update the parameters iteratively to minimize (or maximize) a certain evaluation function. The computation of an approximate electric or magnetic field is done by the FDTD method as a forward solver. The optimization process can be carried out by using many different algorithms such as the Newton method, conjugate gradient method, or evolutionary algorithms. One major limitation of using parametric algorithms is the time of calculation that can be prohibitive for 3-D problems.

In the next section, the nonparametric approach will be briefly described.

\section{NONPARAMETRIC APPROACH}

To improve the interpretation of the GPR assessment a reverse-time migration technique [1] to find the exact location of the targets in heterogeneous media was implemented. The development of this algorithm is based on the notion of a matched filter, which is used extensively in radar applications. Using this algorithm, an image can be perceived as a back propagated wave-field reconstruction of the dielectric contrast within the host medium [1]. The final migrated data for a bistatic configuration can be obtained by the following equation:

$$
S(\mathbf{r})=\left.\sum_{n=1}^{N} \sum_{m=1}^{M} \mathbf{E}_{m n, b p}(\mathbf{r}) \otimes \mathbf{E}_{i n c}(\mathbf{r})\right|_{t=0}
$$

where the subscripts $m$ and $n$ denote the field due to the $m$ th transmitter and $n$th receiver. The bistatic algorithm requires propagation of both the incident and back-propagated fields. Only one antenna is used to send the pulse.

The implementation in FDTD is accomplished by propagating the incident field in reverse while simultaneously propagating the back propagated field forward. As an example, consider the FDTD scenario consisting of four cylinders (with different radii), conductor (1), air (2), and water (1), buried in concrete. The raw data is collected at 31 receiver locations separated by $7.2 \mathrm{~cm}$ with the transmitter located above the dielectric slab (Fig. 1). The three traces near the transmitter were zeroed out since the receiving and transmitting antennas were in too close proximity. The final image occur where the incident field intersects the backpropagated field.

The backpropagated field is the reference field simulated with a nonhomogeneous medium. The matched filter used an homogeneous medium with $\epsilon=6$ and $\sigma=1 \mathrm{mS} / \mathrm{m}$. Three simulations with the incident field in the dielectric's far left, right and center were done.

Fig. 2 shows the final image obtained at $t=0$ that provides a more exact location of the inclusions positions. This process improves the final images provided by the radar inspection data. In addition, its implementation is very simple.

However, this algorithm assumes that the background medium is known-a common assumption in many commercial software for the interpretation of GPR data [5]. In addition, in this simulation line sources were used which can create a problem when the objective is to find targets in close proximity. In real problems, this algorithm can be used for locating conductors and buried pipes but it cannot find the exact characteristics of the inclusions. 


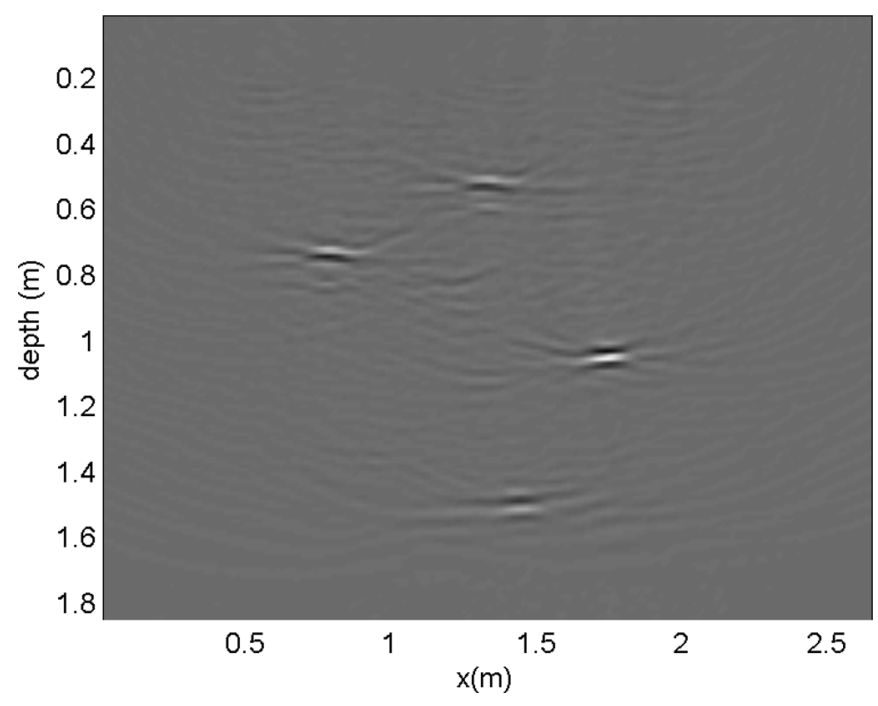

Fig. 2. Bistatic reverse-time migration; final data.

\section{PARAMETRIC APPROACH}

An incident wave and a scattered wave can be used to characterize the scattering object. Usually, in real-world problems, the incident and scattered waves are known and it is desired to identify the scattering object. Therefore, it can be written as an optimization problem involving the scattered wave of the unknown object $\mathbf{E}\left(\theta_{0}\right)$, the reference object, and the scattered wave of a test object $\mathbf{E}(\theta)$. Thus, $\theta^{*}$, the optimum $\theta$, is the argument that minimizes the error of the reference object scattered wave $\mathbf{E}\left(\theta_{0}\right)$ relative to the test object scattered wave $\mathbf{E}(\theta)$. Mathematically

$$
\theta^{*}=\arg \min f(\theta)=\sum_{i=1}^{n s}\left(\mathbf{E}\left(\theta_{0}\right)-\mathbf{E}(\theta)\right)^{2}
$$

where $n s$ are the sample points where the scattered wave is measured. Note that $E\left(\theta_{0}\right)$ is known even though $\theta_{0}$ is unknown-it is the measured value on the receiver antennas.

The scattered $\mathbf{E}(\theta)$ is then generated assuming one test $\theta$, and the optimization procedure aims at minimizing the error between $\mathbf{E}\left(\theta_{0}\right)$ and $\mathbf{E}(\theta)$ in such a way as to identify the scatter object $\theta_{0}$. This paper assumes that $\theta$ and $\theta_{0}$ are made of a set of cylindrical inclusions; thus, $\theta=\left\{\left(r_{1}, c_{1}, e_{1}, \sigma_{1}\right), \ldots,\left(r_{n}, c_{n}, e_{n}, \sigma_{n}\right)\right\}$.

The parametric approach can robustly define the number of inclusions $n$ and their centers $c_{i}$, but it cannot find the other variables. This paper uses the parametric approach to define the radii and the physical properties of the inclusion and, afterwards, find the other properties using the parametric approach.

The problem described in (2) is usually multimodal, as shown in Fig. 3, where the unknowns are the radii of two inclusions given their physical properties. This multimodal characteristic motivates the use of a stochastic approach instead of a deterministic one. This problem was solved here using the PSO, and is described in the next section.

\section{A. Particle Swarm Optimization}

PSO is one of the latest evolutionary optimization methods inspired by nature [2], [3] that includes evolutionary strategy

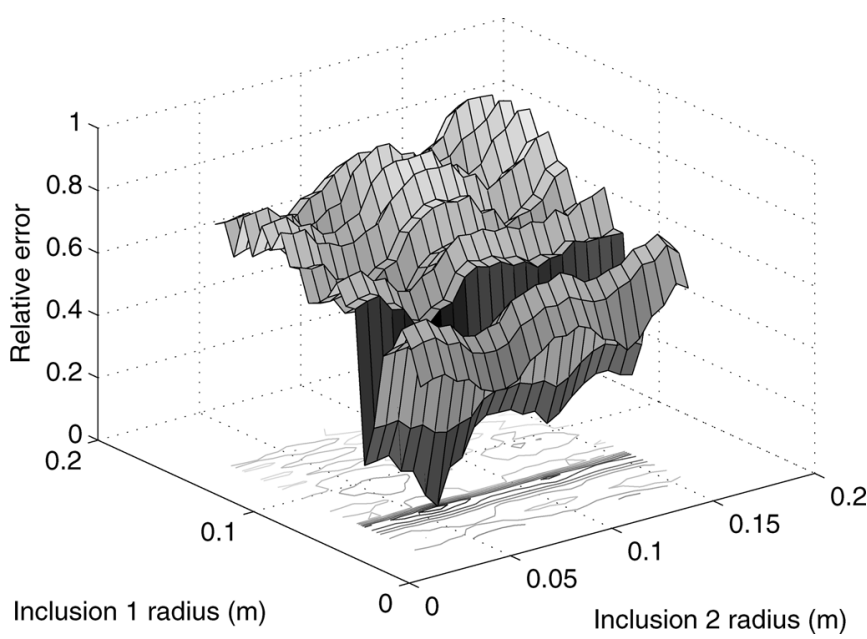

Fig. 3. Surface of (2) considering the problem of finding the radii of two inclusions, given that its location and physical properties are known. Even in this simple example, the surface is clearly multimodal; thus, stochastic methods must be employed in the parametric approach.

(ES), evolutionary programming (EP), genetic algorithm (GA), and genetic programming (GP). PSO is based on the metaphor of social interaction and communication such as birds flocking and fish schooling. PSO is distinctly different from other evolutionary-type methods in a way that it does not use the filtering operation (such as crossover and/or mutation), and the members of the entire population are maintained through the search procedure so that information is socially shared among individuals to direct the search towards the best position in the search space.

The original intention of the method was to graphically simulate the choreography of a group of birds. In a certain moment of the model evolution it was noticed that the algorithm was in fact an optimizer. Using an empirical process many parameters were eliminated, the ones relevant to the social model but not to the optimization, resulting in a simple algorithm.

In a PSO algorithm, each member is called a particle, and each particle moves around in the multidimensional search space with a specific velocity. According to the global neighborhood, each particle moves towards its best previous position and towards the best particle in the whole swarm.

The PSO is similar to GAs due to the random initialization. The first difference is that each potential solution is called particles, instead of individuals, and they "fly" on the search space. To each particle of the swarm during the iterations, the positions of the best solution found to a given particle, called pbest (particle best) is saved.

The best value found considering all the particles is also saved, and is called gbest (global best). At each iteration, the PSO is based on the change in the particle's velocity in the direction of its pbest and gbest, weighted by random terms. The PSO algorithm can be described as follows.

1) Initialize the swarm of particles with random positions and velocities.

2) For each particle calculate the objective function.

3) Find pbest.

4) Find gbest. 
TABLE I

INVERSE SCATTERING INCLUSION DEFINITIONS

\begin{tabular}{lccc}
\hline & Center(x,y) [m] & Radii [cm] & Material \\
\hline Inc 1 & $(0.78,0.84)$ & 6.6 & Air \\
Inc 2 & $(1.32,0.60)$ & 9.0 & Conductor \\
Inc & $(1.74,0.60)$ & 9.6 & Water \\
\hline
\end{tabular}

TABLE II

NUMBER OF FDTD EVALUATIONS SPENT ON AVERAGE, TAKING INTO ACCOUNT TEN TESTS, By THE HYBRID APPROACH PROPOSED IN THIS WORK COMPARED WITH THE PSO ALONE

\begin{tabular}{ccc}
\hline Num. of Inclusions & Hybrid & PSO \\
\hline 1 & 41 & 670 \\
2 & 475 & 3800 \\
3 & 2100 & $N A$ \\
\hline
\end{tabular}

5) Change the velocity and position of each particle according to (3) and (4)

$$
\begin{aligned}
v= & v+c_{1} * \operatorname{rand} *(\text { pbest }-x) \\
& +c_{2} * \operatorname{rand} *(\text { gbest }-x) \\
x= & x+v .
\end{aligned}
$$

6) Return to step 2 until a stop criterion is achieved.

The velocity of each particle in each dimension is limited by a maximum velocity, $V \max$. The acceleration constants $c_{1}$ and $c_{2}$, represent the tradeoff between the search in the direction of pbest and gbest. Usual values for $c_{1}$ and $c_{2}$ are equal to 2 , whereas $\operatorname{Vmax}$ is between $10 \%$ and $20 \%$ of the variable range in each dimension.

\section{RESULTS}

The proposed hybrid algorithm was tested in the inclusion definitions given a nonhomogenous medium with standard deviation $s d=0.25$. The inclusions are defined in Table I. The search space assumes radii $=\left[\begin{array}{ll}5 & 10\end{array}\right] \mathrm{cm}$ each inclusion could be: air, conductor, or water.

The hybrid approach that combines the MFBRT with PSO is compared with the PSO alone considering problems with one, two, and three inclusions. Table II presents the results in terms of the number of function evaluations, taken of a average of 10 simulations, to define the inclusion.

The results show that the number of function evaluations decrease expressively when the approach proposed in this paper is applied compared to the traditional parametric approach. It is important to remark that the MFBRT alone could not solve the whole problem alone.

For instance, in a problem with two inclusions, the PSO has to find four variables, while the hybrid approach proposed in this work allows the PSO to operate with only two. This makes the search more efficient and, therefore, faster. Another remarkable fact is that the cost associated strongly increases as the number of inclusions increases.

This fact limits the use of our approach in situations where the number of inclusions is high. For the pure PSO approach the number of function evaluations diverges with only three inclusions, and it is marked as Not-Available, NA, in Table II.
However, the large search region outlined in this problem makes solving the inverse problem difficult. Although iterative optimization approaches take longer computing times, these approaches give much better image qualities for high-contrast objects than linear inversions such as a diffraction tomography [6], [7].

\section{CONCLUSION}

This paper presented a discussion of the strengths and weakness of parametric and nonparametric approaches for the inverse scattering problem in a nonhomogenous host medium. The nonparametric approach is a robust and fast technique to define the number of inclusions and their centers, but incapable to define their geometry and physical properties.

The parametric approach can solve the general problem but is more expensive in computational terms. However, the use of a stochastic algorithm is necessary because the estimation of inclusions in a large search region with limited-view measurements often fails trapped in a local minimum.

These two approaches are combined in such a way as to try to take advantage of their strengths to decrease the computational burden. This combination was done in two phases: 1) the nonparametric approach is used to define the number of inclusions and their centers; and 2) the parametric approach is used to define the other unknown variables, the inclusions radii and the physical properties. This combination could deliver the characterization with a fraction of functions evaluations when compared to the parametric approach used alone. This approach yields accurate detection of positions and effective physical properties of the targets.

The choice of PSO in the parametric solution was due to the multimodality of the problem that indicates that stochastic techniques must be preferred. As a future research, it is important to find effective ways to decrease the computational effort as the number of inclusions rises. The nonparametric approach is not dependent of the number of inclusions; however, the parametric presents problems as this number rises.

\section{REFERENCES}

[1] C. J. Leuschen and R. G. Plumb, "A matched-filter-based reverse-time migration algorithm for ground-penetrating radar data," IEEE Trans. Geosci. Remote Sens., vol. 3, no. 5, pp. 295-326, May 2001.

[2] R. C. Eberhart and J. Kennedy, "A new optimizer using particle swarm theory," in Proc. Sixth Int. Symp. Micro Mach. Human Sci., 1995, pp. 39-43.

[3] J. Kennedy and R. C. Eberhart, "Particle swarm optimization," in Proc. IEEE Int. Conf. Neural Netw., 1995, vol. 4, pp. 1942-1948.

[4] D. J. Daniels, Ground Penetrating Radar. London, U.K.: Inst. Electr. Eng., 2004.

[5] GSSI Handbook for Radar Testing of Concrete. North Salem, NH: Geophys. Survey Syst., Inc., 2001, p. 13.

[6] H. Jia, T. Takenaka, and T. Tanaka, "Time-domain inverse scattering method for cross-borehole radar imaging," IEEE Trans. Geosci. Remote Sens., vol. 40, no. 7, pp. 1640-1647, 2002.

[7] R. Demining and A. J. Devaney, "Diffraction tomography for multimonostatic ground penetrating radar imaging," Inv. Probl., vol. 13, pp. 29-45, 1997.

Manuscript received June 24, 2007; revised November 1, 2007. Corresponding author: X. L. Travassos (e-mail: lucast@cimatec.fieb.org.br). 